\title{
Flumes for benthic ecologists: theory, construction and practice
}

\author{
D. K. Muschenheim, Jonathan Grant \& Eric L. Mills \\ Department of Oceanography, Dalhousie University, Halifax, Nova Scotia B3H 4J1, Canada
}

\begin{abstract}
Although the utility of laboratory flumes in benthic ecological research has been demonstrated by numerous studies there are no general references on the technique, and the details of flume design have often been neglected in published work. Details of boundary-layer flow relevant to benthic processes and their study in flumes are presented, using a newly constructed flume at Dalhousie as an example. The Dalhousie flume is a straight flow-through channel $3 \mathrm{~m}$ long, with a corewell for sediment samples and adjustable exit weirs to control flow rate and water depth. This design is suitable to assess the stress imposed on organisms and sediments by the motion of fluid near the bottom. The important criteria for channel design, flow visualization and measurement are presented for this and more complex recirculating designs. We report various means of measuring concentrations of suspended particles, allowing calculation of the horizontal flux of organic matter at the bed. Controlled flow experiments are essential to determine the effect of organisms on sediment stability through biofilms or roughening of the sea-bed. The role of the horizontal transport of detrital organic material will become clearer as this technique is more frequently applied in quantitative benthic ecology.
\end{abstract}

\section{INTRODUCTION}

The flow of water over the bottom distributes sediments and food particles for benthic animals. Both the morphology of these animals and their means of acquiring food are adaptive responses to the fluid medium in motion (Rubenstein \& Koehl 1977, Vogel 1981, Nowell \& Jumars 1984). Increasing attention has been paid to the role of horizontal advection in supplying food to benthic organisms (Miller et al. 1984, Wildish \& Kristmanson 1984, Grant 1985); this requires knowledge of the horizontal transport rates of organic detrital materials. Due to the inherent variation in size and density of naturally occurring organic-mineral aggregates, it is impossible to obtain calculated settling velocities from theory, and Ineasured settling velocities can only be approximations with poorly sorted sediments.

Studies with laboratory flumes have been able to isolate flow effects and elucidate relations that could not be detected in field studies or by theoretical approaches, For example, Wildish \& Kristmanson (1979, 1984) showed strong correlations between the rate of seston input and the growth of suspensionfeeding bivalves. Carey (1983) showed that local scour due to the hydrodynamic effects of worm tubes pro- vided a source of resuspended food particles. Looking more directly at the horizontal transport of such particles, Fisher et al. (1979) measured critical erosion velocities for organic detrital particles from streams and found them to adhere well to the standard Shields 'competency' criterion, formulated for well-sorted inorganic sediments. Grant et al. (1982) used the same criterion as a measure of the way biological processes modified non-cohesive sediments. Our work on the horizontal flux of suspended organic material shows that a substantial gradient exists very close to the seafloor (Muschenheim 1984, Muschenheim et al. unpubl.). The conditions that determine whether the flux maximum occurs at the boundary or a few centimeters above will have important implications for foraging in suspension- and deposit-feeding benthos. Other modelling and experimental efforts have attempted to determine how the food supply to deposit-feeders is mediated by the local hydrodynamic regime (Jumars \& Nowell 1984, Miller et al. 1984).

In addition to the effects of flow on feeding and food supply, benthic organisms have a role in determining the stability of the seabed. This effect has been studied in a flume by Neumann et al. (1970) in tropical sediments, by Rhoads et al. (1978) using natural sediments from Long Island Sound, and by Young \& Southard 
(1978) using Buzzards Bay sediments, Later work (Eckman et al. 1981, Nowell et al. 1981, Grant 1983, Taghon et al. 1984, Eckman \& Nowell 1984) has been directed at separating direct biological effects from biologically mediated hydrodynamic effects on sediment stability. The ability to quantify biological effects on the physical environment, in terms of roughening of the bed or the binding of sediments, has allowed ecological information to become relevant to more applied disciplines, such as engineering geology and fluid mechanics.

Several technological factors have increased the number of recent flume studies. Relatively inexpensive means of measuring flow at fine scales have been presented in the biological literature (LaBarbera \& Vogel 1976, Vogel 1981). As in other disciplines, the advent of micro-computers and appropriate software has made the logging and processing of large sets of data accessible to investigators who otherwise would not have the means to undertake this research.

Despite the experimental appeal of laboratory flumes, direct measurement of both bedload and suspended load in sediment transport studies has been a difficult problem. Bedload motion and particle flux have been experimentally examined from a sedimentological perspective for nearly a century since the work of Deacon (1894, cit. in Gilbert \& Murphy 1914). Studies of the distribution of suspended load started later, with the theoretical formulation of Rouse and experiments by Vanoni (1946) and others. These investigations concern themselves primarily with the transport of well-sorted, non-cohesive inorganic sediments and therefore omit the components of greatest interest to students of the benthos, the organic and organicmineral aggregates in natural sediments.

To be able to manipulate natural sediments and organisms for this type of study requires equipment with special capabilities. These include the ability to (a) simulate natural near-bottom flow conditions while maintaining organisms in life position, (b) observe flow over intact sediment samples with undisturbed organic aggregates and films, (c) introduce and rapidly mix particles into suspension, and (d) allow access for measurement and manipulation. The laboratory situation is ideal, because in the field the structure of flows and their organic particle load are difficult to characterize, especially in unsteady flows (e.g. over tidal flats) or on scales of interest to biologists $(\mathrm{mm}$ to $\mathrm{cm}$ ).

Our concern here is with laboratory flow channels intended to control water flow and the boundary layer processes relevant to benthic animals. The ecological literature includes many contributions dealing with flow, its measurement and the implications of water motion near a boundary, but the details of flume design and operation are dealt with only in a cursory way. Although flumes and flow tanks are obviously adaptable to ecological problems, details of the technology are scattered through a complex literature. The only reference specifically on flumes (Williams 1971) is directed at sedimentologists and is not generally available. Flumes have often been used to study the behaviour of stream insects (Peckarsky 1983) but these experiments are not concerned with boundary layer phenomena. To the marine ecologist aspiring to operate a flume, the prospect of approaching an arcane technical literature can be daunting. The purpose of this paper is to provide constructive information on flume design, operation, instrumentation, methods, and measurement from a benthic ecologist's perspective. We present a newly installed flume at Dalhousie University as an example of a method that has great promise for experimental benthic ecology and that has already yielded significant results.

\section{Design of the Dalhousie flume}

We will use the Dalhousie seawater flume and its instrumentation to illustrate critical aspects of design and operation. Its design is simple, with basic similarities to many flumes now in use, and is compared with other configurations to evaluate their relative strengths and weaknesses

The Dalhousie flume (Fig. 1) is a flow-through channel (non-recirculating) with a $3 \mathrm{~m}$ long working channel, constructed from $1 / 2$ inch $(12.5 \mathrm{~mm})$ acrylic sheet (plexiglas). Channel width is $35 \mathrm{~cm}$ and maximum flow depth is $20 \mathrm{~cm}$. The walls are joined to the flume bed with plexiglas solvent (dichlormethane) and caulked with a thin bead of silicon cement. Cross braces add to the support of the walls (Fig. 1). One inch (25 mm) thick laminated particleboard, resting on cinder-blocks, supports the flume and provides a downchannel slope of between 1 and $2^{\circ}$. A grid of $1 \mathrm{~cm}$ squares placed beneath the clear flume floor aids in instrument positioning and examination of flow characteristics. An opening in the flume floor accepts a flush-mounted $25 \times 25 \mathrm{~cm}$ acrylic corebox which is used to maintain benthic organisms and sediments in the controlled flow.

From a biological point of view, it is critical that the flume materials not be toxic to organisms, that the walls of the working section be clear for observation and photography, and that the channel be open and accessible for measurements and manipulation. For the first 2 considerations clear acrylic sheet is the material of choice, although wooden channels with glass windows have also been used. Obvious benefits are smoothness, visibility, strength and ease of construction, especially sealing. The ease of drilling and tapping acrylic makes machining easier and allows for 


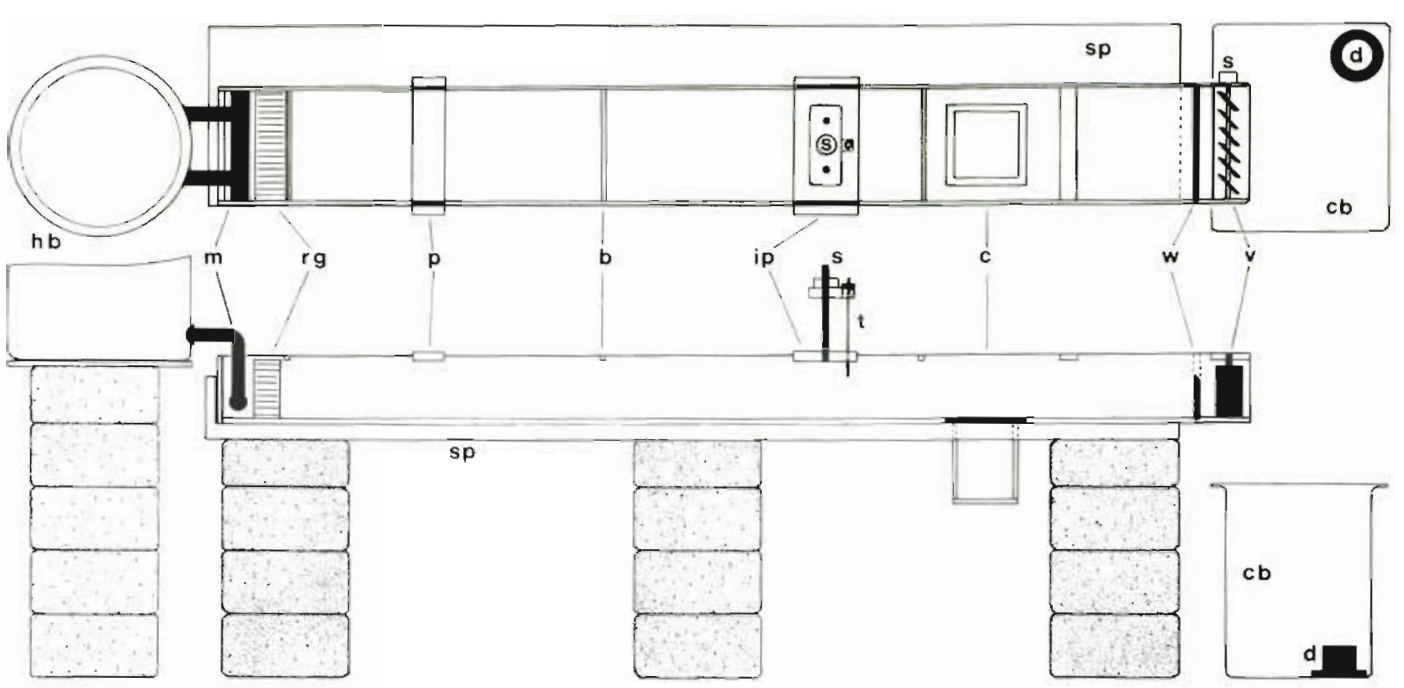

Fig. 1. Plan and side views of the Dalhousie flume, showing the headbox (hb), manifold (m), rectifier grid (rg), moveable platform (p), channel brace (b), instrument platform (ip), with profiling stepping motor (s) and thermistor probe (t). This platform is also moveable, but is generally used in the working section of the channel. The corewell and corebox (c) are located $75 \mathrm{~cm}$ upstream of the sharp-edged weir plate (w) and stepping motor (s) cantrolled weir vanes (v). Also shown are the flume support (sp), catch basin (cb) and drain (d). Not shown are the flow control valves on the manifold and the constant head overflow pipe between the headbox and catch basin. The total channel length is $325 \mathrm{~cm}$

better fit than with other materials. Wooden flumes have also been used successfully, offering benefits in cost, but are difficult to seal, and require the mounting of glass or acrylic windows flush with the inner channel surface. Lastly, moveable instrument platforms should span the channel and at least 1 wall of the flume should be flush with the support to permit photography at bed-level.

Filtered seawater for our flume comes from Dalhousie's Aquatron (Balch 1978) at a constant delivery rate of $450 \mathrm{lmin}^{-1}$. This is sufficient to maintain a constant flow rate of $30 \mathrm{~cm} \mathrm{~s}^{-1}$ at $7 \mathrm{~cm}$ depth and obviates the recirculation of flume water, greatly simplifying flume design and construction. Aquatron water is filtered through sand beds before entering a $500 \mathrm{l}$ capacity head-tank with an overflow pipe to maintain static pressure upstream (Fig. 1). The inflow to the tank can be fitted with a $62 \mu \mathrm{m}$ plankton net to remove additional particles. Water exits the head-tank through a twin-valved ABS plastic manifold, into the flume turbulence chamber. A flow-through flume which receives filtered seawater is essential in organic resuspension or deposition experiments because the flume water supply does not contribute to the particle load.

Entrance and exit conditions. Both the entrance and exit conditions are important to development of the flow. At the entrance the primary concern is to dissipate the large-scale turbulence produced by the pumping (or other) water supply system. The pumped water is introduced through a perforated manifold into a combination of baffle elements, called rectifier grids, just before the entrance. In the Dalhousie flume, flow is rectified by several thicknesses of nylon furnace baffling and a $10 \mathrm{~cm}$ thickness of laminated industrial lighting diffuser. Any installation requires time and testing to develop an efficient combination for a variety of flow conditions. The objective is to force the flow into the channel with a uniform velocity distribution in both the lateral and vertical dimensions, allowing the boundary layer to grow with distance downstream. This can be checked using flow visualization (see below).

The exit conditions, along with supply rate and channel tilt, determine the depth of flow. The Dalhousie flume contains both a simple slotted weir bracket and an articulating vaned weir, operated by a stepping motor, which allows precise positioning and good position-holding torque (Fig. 1). The vanes can be rotated in small increments. Accurate control of flow is essential when determining critical erosion velocity of sediment and detritus.

Fluid engineering texts (e.g. Roberson \& Crowe 1980 , Streeter \& Wylie 1979) present equations for the calculation of flow depth for specific channel and flow characteristics. If relatively slow but deep flow is desired, it will be necessary to impede the flow at the exit. The simplest way is to use a sharp-edged weir spanning the channel, as in the Dalhousie flume, producing a 'backwater effect'. This forces the water to back up and flow over the weir edge, with disruption of flow streamlines for some distance up-channel. In addition, there will be a water surface 'dropdown' extending upstream. Engineering texts recommend 
making flow measurements at least 5 weir depths upstream (Streeter \& Wyllie 1979) to ensure unaltered flow. If a slotted bracket is cut into the channel walls, weirs of different heights can be inserted to maintain a specific depth of flow. A variety of alternative designs, such as articulating vanes, perforated plates or variable width slots have also been used in place of weirs. In any case, one should allow enough channel length so that measurements can be made in a fully developed boundary layer sufficiently far upstream of the exit conditions to avoid their effects.

Flume geometry. In addition to the control of flow velocity, an important concern in designing a flume is boundary layer development. For the range of velocities anticipated there must be sufficient length of channel to develop a boundary layer of workable thickness. Vogel (1981) has emphasized the inverse relation between the Reynolds number and boundary layer thickness; both he and Schlichting (1967) present formulae for this calculation as a function of downstream distance from the leading edge (flume entrance). Nowell \& Jumars (1984) have discussed the implications of this for ecological work such as settling plate experiments.

In straight channels the flow is usually driven by the energy available from the water height differential between the upstream and downstream ends. This differential is often augmented by tilting the flume. For critical measurements of bed shear stress the ideal condition is for the flow to be driven by tilting so that the water depth, and thus the pressure distribution, is equal along the length of the channel. This point is not of sufficient importance to biologists to warrant the trouble involved. What is to be avoided is 'adverse slope', where the channel exit is above the entrance (Chow 1959).

The fact that boundary layers develop on both the channel floor and sidewalls means that to avoid flow artifacts, channel width and wall smoothness are crucial concerns. Calculation of wall boundary layer thickness is not straightforward, due to interactions with the bottom and the free surface. Recommendations for the ratio of flume width to boundary layer thickness (or flow depth if the boundary layer intersects the free surface) vary from 3 to greater than 10. Nowell \& Jumars (1984) suggest that the ratio must be greater than 7 , although most workers seem to take 5 as a minimum value. The only systematic study of flume wall effects (Williams 1970) concluded that width had very little effect on the relation between bulk flow and net sediment transport, but that narrow flumes produced significant artifacts in bedforms. He did not address the effect of width on the shape of the velocity profile. The $35 \mathrm{~cm}$ width of the Dalhousie flume allows a maximum flow depth of $>5 \mathrm{~cm}$ for most work, while the $20 \mathrm{~cm}$ wall height allows greater depth at slower flows. Narrower flumes, especially smoothwalled ones, can still be of use but one must ensure that the bottom boundary layer has developed in an unimpeded fashion (e.g. Eckman \& Nowell 1984). Some workers (Wildish \& Kristmanson 1979, 1984) have flared the channel walls with distance downstream to provide more free bottom area, but this presents problems due to flow deceleration; the bed shear stress will change constantly down channel because the boundary layer never reaches equilibrium. This was of little concern in their work, but it should be avoided in work involving sediment transport.

Working section. The primary concern of a benthic ecologist using flumes is to be able to maintain organisms or place intact field cores in a controlled flow. Rhoads et al. (1978) used an ingenious arrangement with a small closed-channel flume, whereby part of the channel was a removable, diver-deployed corebox. The working section of the Dalhousie flume consists of a $25 \times 25 \mathrm{~cm}$ corewell (Fig. 1), positioned $2.25 \mathrm{~m}$ down-channel (far enough upstream of the exit to experience undisturbed flow), which accepts an $18 \times$ $18 \mathrm{~cm}$ boxcore. In the field, the boxcore is inserted into the sediment, the surrounding sediment excavated, and a bottom plate secured in place with elastic bands. A number of these cores can be obtained from the field on a single day. The corewell in the flume has an adjustable false bottom to accommodate variation in core height. Once the boxcore is in the corewell, the flume can be filled slowly allowing gentle filling of the core. At this point the sides of the box can be removed leaving an undisturbed sediment sample. This technique is ideal for work with ripples, tubes, fecal mounds, etc. The grain roughness characteristics of the core can be simulated by spreading a thin layer of ashed sediment upstrean. For other studies the corewell can be covered with an acrylic plate, converting the channel into a planar bed.

\section{FLOW MEASUREMENT}

\section{Theory of near-bottom flow}

There are 2 fundamental consequences of living at a solid-fluid boundary that differentiate the benthos from the plankton. The first is the presence of a substratum providing physical support and sedimented detrital particles serving as a food source. The second is the existence of a near-bottom velocity gradient, the 'boundary layer', where the drag of the bottom retards the fluid for some distance above the substratum. The velocity diminishes to zero at the substratum. Within the boundary layer, the differential velocity produces a 
shearing force or shear stress which resuspends and transports sediments and organisms. Differential redeposition of particles results from hydrodynamic sorting of organic-rich and inorganic sediments. The nearbottom water chemistry, microbial environment and pore-water conditions (Berner 1974, Jumars \& Nowell 1984, Jorgensen \& Revsbech 1985) will also reflect the effect of this shear on the bottom.

The stress in a fluid $(\tau)$ is proportional to the change in velocity $(u)$ with height above the bottom $(z)$ multiplied by the fluid's dynamic viscosity $(\mu)$, i.e:

$$
\tau=\mu \frac{\delta u}{\delta z}
$$

From dimensional arguments, fluid dynamicists express this stress as a 'shear velocity', $u_{*}$, the square root of the ratio of the bed shear stress $(\tau)$ to the fluid density $(\varrho)$ :

$$
u_{*}=\left(\tau_{0} / 0\right)^{1 / 2}
$$

Following the arguments outlined in the 'Appendix', for a large variety of flow conditions the mean velocity profile will be a function of the magnitude of the flow as well as the roughness of the boundary. The profile is often expressed in logarithmic form as:

$$
u_{z}=\frac{u_{*}}{x} \ln \frac{z}{z_{0}}
$$

where $x=$ von Karman's constant and $z_{0}=$ roughness length (see 'Appendix'). Given velocity measurements at different heights within the log-layer, the bed shear stress, $\tau_{0}$, can be calculated from the slope of the velocity profile. More detail about the structure of the boundary layer may be found in Middleton \& Southard (1978) and Nowell \& Jumars (1984).

Roughness of the boundary plays an important role in determining the shape of the velocity profile. The $z_{0}$ term parameterizes only roughness that affects the flow through skin friction drag, e.g. viscous forces around sand grains. This is also the easiest situation to handle in flumes. As the 'roughness elements' (sediment grains, pebbles, fecal mounds, worm tubes, etc.) get larger and farther apart they start to affect the velocity profile through form drag in addition to skin friction, and the observed profile will actually be a composite of the 2 effects (Arya 1975, Chriss \& Caldwell 1982. Nowell \& Jumars 1984). Local flow effects become more complex, with flow separation, horseshoe vortices and internal boundary layers forming in the lee of objects. For details of these flow patterns see Taylor \& Dyer (1976), Middleton \& Southard (1978), Paola (1983) and Eckman \& Nowell (1984). Although individual roughness elements direct flow toward the bed and promote scour near them (Eckman et al. 1981,
Eckman \& Nowell 1984), their main effect on the mean flow is turbulence farther from the bed. At certain numerical densities the interaction of the turbulent wakes of individual roughness elements causes new flow patterns to emerge (Nowell \& Church 1979).

\section{Methods}

In the course of our flume studies, several simple, inexpensive approaches to sampling and calibration problems were developed. Some have since been superseded by more technical approaches, but the value of simplicity makes them worth considering. The simplest and most intuitively satisfying approach to quantifying fluid flow is to add dye or discrete particles to the water and follow their paths along streaklines. The assumptions in this Lagrangian approach are that the particles have the same momentum as the fluid and, if they are small, that they are nearly neutrally buoyant. If highly turbulent flow is required, turbulent diffusion will make the markers difficult to follow in addition to requiring many more measurements to extract a reliable mean value. Several dyes (red food colour, potassium permanganate, fluorescein) have been used effectively in flow visualization but care must be taken to match the buoyancy characteristics of the dye and the fluid. Dilute solutions (or suspensions) maintained at flume water temperature are most effective.

A simple arrangement for visually measuring velocity profiles involves mounting identical transparent grids on opposite sides of the flume and suspending a pipette full of dye above the center of the channel. Velocity at a particular height is measured by releasing a drop of dye just upstream of the starting mark on the grid, then timing it as it travels the length (10 or $20 \mathrm{~cm}$ ) of the grid, ensuring that it remains at the same height. Runs are repeated until enough replicates have been obtained to give a reliable mean value. The method is tedious but accurate for mildly turbulent flows up to $10 \mathrm{~cm} \mathrm{~s}^{-1}$. Care should be taken to ensure that there is no cross-channel transport. Vertical resolution depends on channel width, but it is usually accurate to within $2 \mathrm{~mm}$. A typical velocity profile taken by this method is presented in Fig. 2. The loglinear regression coefficient is 0.999 for the points in the log layer and calculations with Eq. (3) indicate that $\mathrm{u}_{*} \sim .05 \mathrm{u}$, indicating a fully developed boundary layer (Tritton 1977).

Although we have used flow visualization, our usual method of flow quantification is thermistor anemometry. Thermistors have a coefficient of resistance with temperature of about $-4 \%\left(\mathrm{C}^{\circ}\right)^{-1}$ (Horowitz \& Hill 1980), thus their incorporation into the appropriate 


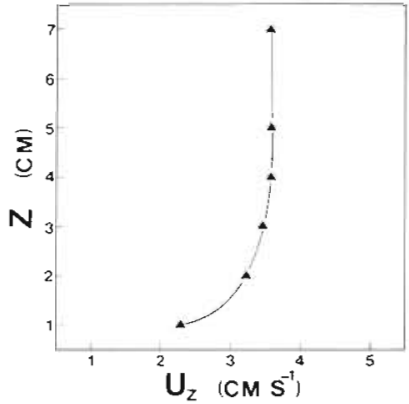

Fig. 2. Profile of velocity $\left(u_{z}\right)$ vs height above bottom $(z)$, taken in the flume by flow visualization with dye streams. The linear regression of velocity on $\log$ (height above bottom) yields a regression coefficient on log (height above bottom) yields a regression coefficient of 0.999. Boundary-layer thickness in this case was $3.6 \mathrm{~cm}$ and $\mathrm{u}_{*}=0.20 \mathrm{~cm} \mathrm{~s}^{-1}$. The procedure is described in the text

circuitry can be used to measure temperature and convective cooling of the thermistor element as a function of flow. Steinhart \& Hart (1978) and Bennet (1981) have tested various higher-order fitting schemes for the relation of resistance vs temperature for oceanic applications.

In the most common applications the thermistor is used as a variable element in a voltage divider or a resistance bridge. The power dissipated in the thermistor maintains its temperature at 20 to $40 \mathrm{C}^{\circ}$ above that of the fluid. The thermistor's resistance is known through calculation from the circuit output. Due to variation in the thermal boundary layer around an individual sensor, each must be calibrated, paying attention to the thermistor's angular response. Glasstipped thermistor 'beads' are available in a variety of shapes, with tip diameters as small as .02 cm (Chriss \& Caldwell 1984b). Detailed reviews of thermistor anemometry can be found in Comte-Bellot (1976) and Gust (1982). Vogel (1981) provides a design for a temperature-compensated resistor bridge circuit, as well as names and addresses of suppliers. An analogous method, using a flush-mounted thermistor film, gives a more direct measure of the shear stress at the bed and has been used for measurementes of localized shear stress (Paola 1983). This must be done with care, because sedimentation quickly alters the thermal conductivity of the sensor and affects its calibration.

; Although the components needed for thermistor anemometry are inexpensive, calibration can require substantial time and effort. Numerous schemes exist, from optically monitored rotating tables to variable speed lathes, to move the thermistor through still, unstratified water at a known speed. We have found that the most suitable alternative is to mount the thermistor in a pipe through which water is run at a known velocity.

This can be done either in a turbulent flow, where the pipe flow is measured by timing dye pulses (much as described in the previous section), or, as Vogel (1981) has suggested, in laminar pipe flow. Vogel's method allows use of the Hagen-Pouseille equation to calculate the velocity at the centerline from the discharge rate. The trick is to establish laminar flow in a pipe of large enough diameter that the thermistor will not impede the flow. Since the onset of turbulence is predicted classically to occur at $\mathrm{R}_{e}>2000$, a 1 inch $(2.54 \mathrm{~cm})$ diameter pipe limits laminar flow to velocities under $\sim 8 \mathrm{~cm} \mathrm{~s}^{-1}$. Fortunately, methods for extending the range of laminar flow are well known. At Dalhousie we have been able to maintain laminar flow up to $R_{e}$ 's of 12,000 using a modified version of Osborne Reynolds' pipe flow apparatus (Fig. 3), which we have incorporated into the flume laboratory. This is done by baffling the supply input to reduce turbulence, flaring the inlet of the calibration pipe and using a long enough smooth-walled acrylic pipe to develop a fully laminar profile. The flow centerline is located from the thermistor output signal, at which point the signal and discharge rate are recorded. Discharge is measured with a stopwatch and graduated glass cylinder. The procedure is repeated through a range of velocities at the same temperature as a flume run.

\section{Data logging and control}

Although thermistor measurements of velocity profiles can be read from a voltmeter or strip chart recor-

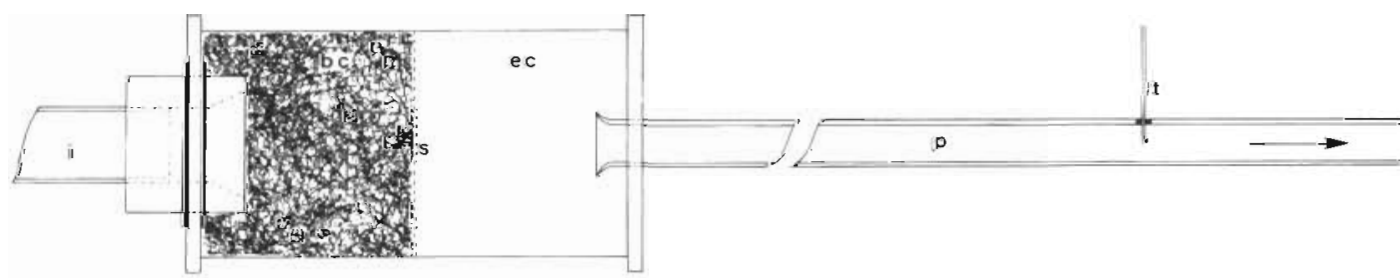

Fig. 3. Reynolds apparatus for thermistor calibration in laminar pipe flow. The arrow denotes the direction of water flow. Water from the flume headbox enters through the inlet (i), passes through the baffle chamber (bc), 2 mm mesh Nytex screen (s) and entrance chamber $(\mathrm{ec})$ before entering the flared inlet to the clear acrylic pipe (p). The thermistor is inserted into the pipe approximately $2.5 \mathrm{~m}$ downstream of the pipe entrance. Total length of the baffle and entrance chambers is $30 \mathrm{~cm}$. The acrylic calibration pipe is $3.3 \mathrm{~cm}$ in diameter (o.d.) 


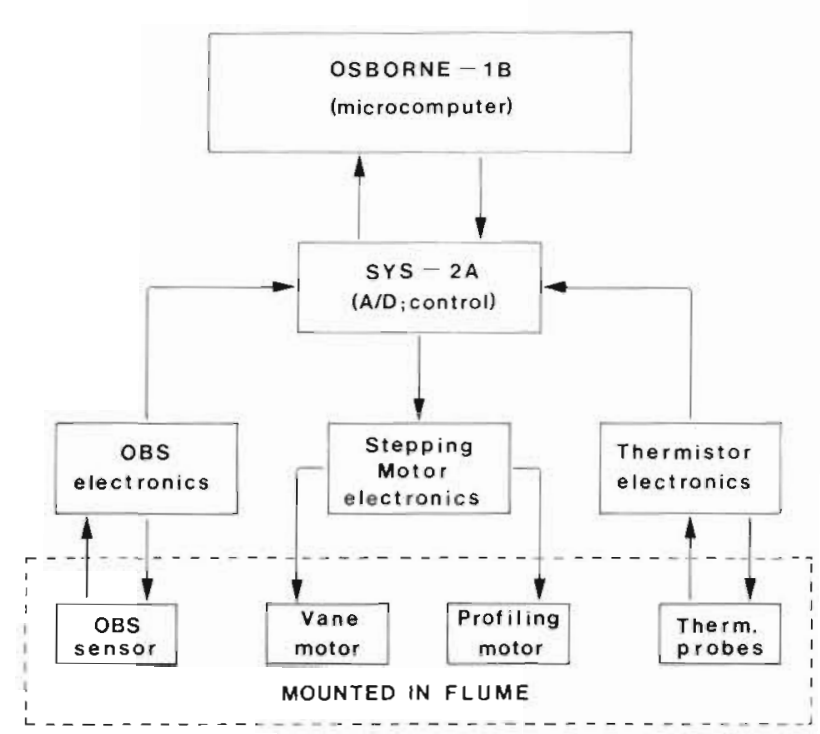

Fig. 4. Schematic of data-logging and control systems used with the Dalhousie flume. See text for details of operation. A/ $D$ denotes analog-to-digital converter. OBS denotes the optical back-scatterance instrument. Arrows indicate the direction of flow of information

der, it is much more convenient (and accurate) to use an electronic data logger attached to a microcomputer. A number of very inexpensive data-logging and microcontroller boards are available, most with resident versions of high-level computer languages (Basic, Pascal, etc.). The flume laboratory at Dalhousie uses an Octagon Systems (Westminster, Colorado) SYS-2A board through the serial port on an Osborne 1-B microcomputer to integrate, digitize, store and process thermistor data (Fig, 4).

Confidence in one's estimate of $u_{*}$ increases with an increasing number of measurements made within the log layer (see 'Appendix'). In the compressed boundary layer in a flume this means making measurements at fine spatial scales. This can be done with a micromanipulator or vernier gauge. We originally mounted the thermistors on the focusing mechanism of a swing-arm microscope for accurate vertical resolution. We now mount the thermistors on a movable carriage regulated by a lead screw and stepping motor (Fig. 4). This system allows vertical steps of $0.1 \mathrm{~mm}$ which can be pre-programmed on the computer to produce automated velocity profiles. Some companies provide ready-to-run controller boards, although the cost is high, often twice that of the separate components.

\section{Other velocity methods}

Various electrochemical techniques are available for measuring velocity, but most involve using fluids toxic to organisms (see Vogel 1981). The most benign and widely used is the hydrogen bubble generator. The application of a $5 \mathrm{~V}$ potential to a very fine cathode wire in seawater will produce bubbles small enough to be nearly neutrally buoyant yet still be visible. Wildish \& Kristmanson (1984) used this technique by timing how long it took the bubbles to fill the gap between the anode and the cathode. Other visualization techniques used with biological systems include milk and denatured egg albumin (Carey 1983).

Mechanical methods such as miniature cup anemometers and deflection of buoyant objects have been used occasionally (Young \& Southard 1978). Neither provides good spatial resolution. A Pitot tube and manometer provide a simple, compact system for directly measuring the dynamic pressure (stagnation pressure-static pressure) at a point in the flow. As long as the flow is uniform (parallel streamlines), steady, and there is no substantial change in surface slope between the 2 pressure ports, the method is direct and accurate at high flow rates. The velocity is obtained by applying the Bernoulli equation; its precision will depend on how sensitive a manometer is used.

Although fine-tipped thermistors disturb the flow relatively little, some devices are totally non-invasive. The most elegant is the Laser Doppler Velocimeter (LDV, Penner \& Jerskey 1973). Another non-invasive technique is high-speed cinematography, provided fine tracer particles (e.g. hydrogen bubbles) are already present in the flow. Using this technique in stereo photogrammetric configuration allows 3-dimensional determination of particle paths and flow but data analysis is time consuming. For a non-biological example of its application see Kent \& Eaton (1982).

\section{SUSPENDED SEDIMENTS}

\section{Theory}

Some problems require knowledge of the concentration of suspended particles in the flow, either as a bulk concentration or as a gradient. The equilibrium concentration profile of particles of a given fall velocity, $\overline{\mathrm{W}}_{\mathrm{s}}$, is given by the Rouse equation (Vanoni 1946, Raudkivi 1976, Middleton \& Southard 1978, Nowell et al. 1981). In its simplest, non-depth-limited form it is:

$$
\mathrm{C}_{\mathrm{z}}=\mathrm{C}_{\mathrm{a}}(\mathrm{a} / \mathrm{z})^{\mathrm{p}}
$$

where $C_{a}=$ reference concentration at a reference height, $a_{i} p=\bar{w}_{s} / x u_{*}$, the ratio of particle settling velocity $\left(\bar{w}_{s}\right)$ to von Karman's constant $\left({ }_{2}\right.$, see 'Appendix') times the shear velocity. The quantity $p$, referred to as the 'Rouse number', is sometimes used as an index of whether sediment of a given fall velocity will travel as suspended or bedload material in a given 
flow. Nowell et al. (1981) applied it to fecal pellets of the polychaete Amphicteis scaphobranchiata to predict that they would travel as bedload under most conditions.

\section{Methods}

A major advantage of a flow-through flume such as ours is that particles can be introduced into it. Particles supplied from a carboy of concentrate (e.g. phytoplankton) are mixed into the flume manifold using a peristaltic pump. This allows upstream-downstream comparisons of particle load, studies of deposition to the bed, or determination of uptake by organisms. The most straight-forward approach to sampling suspended sediment concentrations is to aim a tube into the flow and remove sediment-laden water (Downing et al. 1981, Nielsen et al. 1981, Nielsen 1983). Although few studies (Vanoni 1946, Wildish \& Kristmanson 1984) have attempted to match the velocity $\left(u_{s}\right)$ at the sampling tube to the velocity $\left(u_{a}\right)$ at that height in the flow, large deviations from isokinetic sampling will cause serious artifacts in estimating concentration. Vanoni (1946) suggests that $u_{a}>u_{s}$ will overbias the estimate while $u_{a}<u_{s}$ will underbias it. However, W. D. Grant (pers. comm.) suggests that matching velocity at the sampling tube opening to within $50 \%$ of the ambient flow velocity will avoid serious artifacts.

A siphon sampling array has been used in the Dalhousie flume to sample concentration gradients of natural sediments in suspension. Isokinetic sampling is maintained to within $25 \%$ of the ambient velocity, and a constriction in the tube and conduit (teflon tubing) compresses streamlines to accelerate the flow and minimize settling in the lines. A concentration profile of natural organic-mineral aggregates obtained with this method is presented in Fig. 5, showing a substantial gradient increasing toward the bottom, as would be predicted by the Rouse equation.

Various optical devices can be used to measure suspended sediment concentration. The mounting of a transmissometer on the outside walls is a non-intrusive approach, but it suffers from sensitivity to forward light scattering by bubbles and the integration of effects across the width of the channel, including both walls. In the Dalhousie flume we now use an Optical BackScatterance Sensor (OBS) described in Downing et al. (1981). The back-scatterance of a small volume $(1.3 \mathrm{ml})$ of water is measured at rapid rates. Due to the poorly defined back-scatter angle, Mie scattering theory cannot be applied, so the instrument must be calibrated with suspensions of the sediment being measured. The instrument is sensitive to both organic and inorganic suspensions down to concentrations of a few $\mathrm{mg} \mathrm{l}^{-1}$. It can be used to detect resuspension from cores or parti-

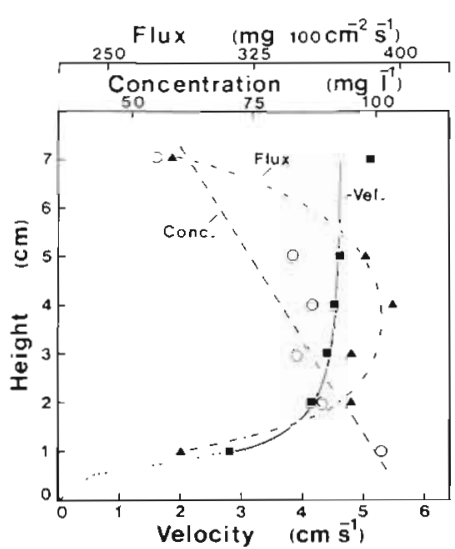

Fig. 5. Vertical profiles of velocity (ロ), suspended particulate matter (SPM) concentration (O), and horizontal flux of SPM (4) for a single flume experiment. Velocity and flux curves were fitted by eye. Concentration curve is a least squares regression $(r=0.9096)$. Water depth was $9.0 \mathrm{~cm}$. Boundary layer thickness was $4.5 \mathrm{~cm}$. Deviation of the $7 \mathrm{~cm}$ velocity point is due to surface acceleration from air conditioning currents in the laboratory. Dashed portion of velocity curve represents the $\log$-linear profile $(r=0.9976)$ extrapolated downward to the height of the calculated viscous sublayer $\left(\delta_{\mathrm{vsl}}=12 \mathrm{v} / \mathrm{u}_{*}=\right.$ $0.5 \mathrm{~cm}$ : see 'Appendix') and a linear profile extrapolated from there to the boundary

cle removal in upstream-downstream comparisons. Test calibrations of the OBS with glacial clays as well as beach sands yield highly linear calibration curves, but the slope and y-intercept are both sensitive to flocculation effects.

\section{Alternative methods}

More complex and expensive equipment includes the LDV which could be used to measure concentrations of suspended material. Another technique used successfully to measure suspended particles is acoustic backscatter. Orr \& Grant (1982) have demonstrated that this technique can detect single sand grains in suspension over ripple beds and Orr \& Rhoads (1982) demonstrated its utility for profiling the top $2 \mathrm{~cm}$ of surface sediments. However it requires sophisticated, expensive equipment and skilled technical help.

\section{Alternative flume designs}

In contrast to our flow-through system, a wide variety of recirculating flumes, from small, annular channels (Taghon et al. 1984) to large 'racetrack'-shaped flumes of $10 \mathrm{~m}$ length, have been used in biological studies. Recirculation saves water, a practical concern where large volume supplies are not available.

Driving the flow in recirculating flumes is complex. 
Taghon et al. (1984) used an annular channel of $2 \mathrm{~m}$ radius and $10 \mathrm{~cm}$ width to study fecal pellet transport and breakdown. The flume was kept level and the flow was driven by the motion of a surface friction plate, in turn rotated by a hydraulic drive in a separate, inner channel. This method allows essentially infinite length for boundary layer development while not forcing the water through a pump, which could destroy particles or organisms. Its disadvantages include the development of a surface boundary layer, complicating the flow pattern, in addition to other flow artifacts common to curved channels. Vogel (1981) presents a design for an impeller-driven recirculating flow tank which has also been used by Carey (1983). Another way of recirculating water is to use a large paddlewheel. This moves large volumes of water but does little damage to particles and organisms traveling in suspension. To minimize turbulence the paddle vanes can be articulated so the pushing surface remains normal to the boundary. Because even the simplest form of paddlewheel requires skilled construction techniques this method is usually reserved for large expensive flumes.

The main drawback common to all flumes with curved channels is the development of secondary circulation patterns. These are characteristically helical flows violating the assumption that the log layer profile develops 2-dimensionally. This makes the calculation of bed shear stresses from velocity profiles impossible. The usual remedy in racetrack-type flumes is to place baffles, by trial and error adjustment in the curved sections of channel, before the rectification step.

\section{FURTHER DESIGN CONSIDERATIONS}

In experiments at high flows (e.g. ripple migration) a flow-through flume of limited length may not have a fully developed boundary layer over the working section. Even with fine vertical positioning of a thermistor, it may be difficult to measure enough points in the boundary layer to determine $\mathrm{u}_{*}$. Under these circumstances, we have used a velocity profile to determine free-stream velocity. The relation between $u$ and $u_{*}$ (Schlichting 1967) suggests that free-stream measurements provide a valuable index of flow, even though shear stress cannot be measured. We emphasize that there are many conditions under which any flume design will be limited; as long as these limitations are considered, valuable experimental results can still be obtained. Even simple observations on the behaviour of organisms and organic particles under normal flow conditions are sorely lacking in marine benthic ecology.

There are also several ways that one can augment the thickness of a flume boundary layer. A common means for doing this is by 'tripping' the boundary layer. This is a technique that has long been used in wind tunnel work (Cermak \& Arya 1970) and can be applied to flumes. The strategy is to use a row of roughness elements (small stones, etc.) that span the channel and create an internal boundary layer, much like that behind an isolated roughness element (Arya 1975, Paola 1983). If the elements are of appropriate size and spacing and are placed near the entrance, the boundary layer will intersect the free surface at some point downstream and the flow will be fully developed from there on. The appropriate size and spacing will depend on flow speed and depth and must be determined by trying different configurations and measuring velocity profiles downstream. A more complex device that shows promise is the 'elliptic wedge generator' of Ligrani et al. (1979, 1983, 1985). It consists of a set of spires and a small barrier placed in the flow. Adjustment of spacing and height will affect the flow properties. The device has been successfully used to create thickened wind tunnel boundary layers with the same momentum and heat flux characteristics as in natural boundary layers.

Flow rectification has already been discussed but an additional point should be made. Depending on the configuration of diffuser material, the flow coming out of the entrance may not exhibit a smooth surface. If visible surface ripples or waves are present, they should be damped out to minimize their interaction with the walls. This is most easily done with a short length of thin plastic or rubber sheet attached to the entrance which is allowed to trail several centimeters downstream on the surface.

\section{APPLICATION OF THE DALHOUSIE FLUME}

Although simple in design, the flume at Dalhousie has been used for studies involving both bedload and suspended load transport of organic detrital particles, as well as the stabilizing effects of microbial films in binding surface sediments.

Grant et al. (in press) used the flume to control flow over cores taken from Eastern Passage, Halifax Harbour. Their experiments showed several characteristic stages of erosional transport, explaining how bedform migration could account for the small-scale patchiness of algal films observed in the field. SEM micrographs of natural and flume samples showed the binding effect of microbial extracellular exudates (from diatoms) and changes in sediment microtopography after erosion. Comparison of biogenic binding effects on calm and stormy days indicated that the films have a significant effect on the exchange of organic matter 
between the sediment and the water column. These studies are continuing with bacterial films.

Experiments by Muschenheim et al. (unpubl.), have assessed the role of the horizontal flux of organic particles in the ecology of benthic suspension feeders. Modelling the vertical profile of particle flux indicated that, depending on the relation of particle settling velocity, shear stress and roughness, the height at which maximum horizontal flux occurs may be the bed surface or several centimeters above it. Flume experiments with natural sediments show the same pattern, when flux is calculated from the product of measured profiles of velocity and sediment concentration (Fig. 5). At high flow rates, suspension feeders must extend farther into the water column to maximize their food intake. Work is continuing to assess the behaviour of a suspension-feeding spionid polychaete (Spio setosa) in response to changing regimes of flow and particle concentration.

\section{SUMMARY AND CONCLUSIONS}

Flumes are essential tools for the quantitative study of biologically important boundary-layer processes. Binding by biofilms and erosion by tube-wake turbulence are only 2 important biological effects on sediment transport that have been elucidated in flume studies. Small-scale feeding studies as well as horizontal organic flux and the growth of organisms are easily controlled and measured in flow channels. These problems, along with many other studies of primary importance in quantitative marine benthic ecology, are amenable to imaginative new applications using flumes

The simplest design - that of a straight, flow-through channel - is ideal for studies of erosion and deposition of organic particles. Losses from or additions to natural core samples can be followed easily by changes in concentration in the sediment and water column. When a long particle residence time or infinite bottom length are required, recirculating designs may be useful, but caution must be taken to account for secondary circulation patterns.

Simple devices are available to measure velocity and suspended sediment profiles. Although time-consuming, they are direct and require no calibration. Inexpensive technological means for velocity measurement and sensor positioning are also available, but they require more time to set up and calibrate. Their advantages are greater precision and ease of use.

Because the boundary-layer must develop, a channel of sufficient length is required if bed shear stresses are to be calculated from velocity profiles. Often, the design of the flume channel will be a compromise between length, available space, and cost. Even relative measures of flow may be appropriate for certain experiments, especially when they are combined with behavioural observations. One may also employ various tricks to create thick boundary layers in relatively short lengths of channel.

As long as the appropriate dimensional scaling holds between the laboratory and natural environment, and the operator remembers that he or she is creating a model of the natural system, flumes allow unique insights into benthic processes. Their potential utility in the study of physical processes in benthic ecology is undeniable, largely because natural flows are complex and difficult to study in the field, even with a battery of expensive and sophisticated equipment. Flumes offer the opportunity to make detailed observations and measurements while controlling the speed with which water moves through the system, the turbulent stress exerted by the fluid on the bottom, as well as the particle load. Important questions of large-scale detritus transport and small-scale (organism-sized) food availability are best addressed with this experimental tool. The measurements may be as simple or complex as required, but they are repeatable and well characterized, unlike the situation in the field. Increasing use of this technique will continue as benthic ecologists become more aware of the importance of horizontal advection in marine ecosystems.

\section{APPENDIX}

Derivation of the logarithmic velocity profile. The form of the velocity profile above a flat boundary is semi-logarithmic. Details of its derivation can be found in most fluid dynamics text (e.g. Tritton 1977). Most simplistically, the stress, $\tau$, in a fluid is related to the velocity gradient through the molecular dynamic viscosity, $\mu$, as shown by Eq. (1). Expressing this in terms of the kinematic viscosity, $v$, yields:

$$
\tau=Q \vee \frac{\delta u}{\delta z}
$$

Where $\mathrm{o}=$ the fluid density; $\mathrm{z}=$ height above the boundary; $u=$ the mean streamwise velocity for larger-scale phenomena, $v$ is replaced by an analogous 'eddy viscosity', $K_{v}$, with units of velocity times length or, in this case, $u_{*}$ times $z$ :

$$
\mathrm{K}_{\mathrm{v}}=x z \mathrm{u}_{*}
$$

where $x=$ a constant (von Karman's constant), characteristic of turbulent wall flows and experimentally determined to be 0.41 under most conditions; $u_{*}=$ shear velocity defined in Eq. (2). The relation then becomes: 


$$
\frac{\delta \mathrm{u}}{\delta \mathrm{z}}=\frac{\mathrm{u}_{*}}{\chi} \cdot \frac{1}{\mathrm{z}}
$$

Since $u_{*}$ and $x$ are constant for any given steady, uniform flow, this integrates to:

$$
\mathrm{u}_{z}=\frac{\mathrm{u}_{*}}{x} \ln (\mathrm{z})+\mathrm{C}
$$

With further dimensional considerations (see Landau \& Lifshitz 1959) the constant of integration is taken to be equal to

$$
-\frac{\mathrm{u}_{*}}{x} \ln \left(\mathrm{z}_{\mathrm{o}}\right)
$$

where $z_{0}=$ a parameter related to bed roughness, so,

$$
\mathrm{u}_{\mathrm{z}}=\frac{\mathrm{u}_{*}}{x} \ln \frac{\mathrm{z}}{\mathrm{z}_{0}}
$$

which is the same as Eq. (3).

Analytically, $z_{0}$ is the $z$-intercept, but the logarithmic relation does not hold very close to the wall, where viscous forces dominate. This region, the viscous sublayer (VSL) is on the order of $12 \mathrm{v} / \mathrm{u}_{*}$ thick, with a linear velocity profile given by:

$$
\mathrm{u}_{\mathrm{z}}=\mathrm{zu}_{*}^{2} / \mathrm{v}
$$

The flow is then called 'hydraulically smooth'. In the ocean, VSL's from a few millimeters to a couple of centimeters thick have been reported from measurements made on the continental shelf off the coast of Oregon (Caldwell \& Chriss 1979, Chriss \& Caldwell 1984a). If the flow is strong enough, or the bottom rough enough, the condition of 'hydraulically rough' flow exists and the VSL is present only in the interstices of the bed.

From arguments presented in Tritton (1977, p. 275) there is no discontinuity between the 2 layers and the stress is assumed to be constant with height this close to the boundary. This means that a stress calculated from logarithmic layer measurements is the same as the stress acting at the bed. Taking a series of velocity measurements and regressing $u$ on $\log z$ will permit calculation of $z_{0}$ and $u_{*}$ and, subsequently, the bed shear stress from Eq. (3). One should note that, for the regression to be statistically significant, there must be enough data points to resolve the profile. In the compressed boundary layers created in flumes, this requires instruments and methods with suitable spatial resolution. More on the calculation of bed shear stress can be found in Middleton \& Southard (1978).

Acknowledgements. The authors have benefited from discussions with D. Carey, T Chriss, G. Gust, W. Grant, and D. Rhoads. P. Jumars and A. Nowell have been especially helpful. D. Conrad and A. Ramard designed and built the control circuits. U. Bathmann, B. Hargrave, D. Huntley and K. Kranck critically read the manuscript. Support for the construction of the flume and its instrumentation came from grants from the Natural Sciences and Engineering Research Council of Canada.

\section{LITERATURE CITED}

Arya, S. P. S. (1975). A drag partition theory for determining the large-scale roughness parameter and wind stress on the Arctic pack ice. J. geophys. Res. 80: 3447-3454

Balch, N. (1978). A description of the pool and tower tanks in the Aquatron laboratory, Dalhousie University. In: Balch, N., Amaratunga, T., O'Dor, R. K. (ed.) Proceedings of the workshop on the squid Illex illecebrosus. Fish. mar. Serv. Tech. Rept. No. 833

Bennet, A. S. (1972). The calibration of thermistors over the temperature range $0-30^{\circ} \mathrm{C}$. Deep-Sea Res. 19: 157-163

Berner, R. A. (1974). The benthic boundary layer from the viewpoint of a geochemist. In: McCave, I. N. (ed.) The benthic boundary layer. Plenum, New York, p. 33-55

Caldwell, D. R., Chriss, T. C. (1979). The viscous sublayer at the sea floor. Science 205: 1131-1132

Carey, D. A. (1983). Particle resuspension in the benthic boundary layer induced by flow around polychaete tubes. Can. J. Fish. Aquat. Sci. 40 (Suppl. 1): 301-308

Cermak, J. E., Arya, S. P. S. (1970): Problems of atmospheric shear flows and their laboratory simulation. BoundaryLayer Meteorol. 1: 40-60

Chow, V. T. (1959). Open channel hydraulics. McGraw-Hill, New York

Chriss, T. C., Caldwell, D. R. (1982). Evidence for the influence of form drag on bottom boundary layer flow. J. geophys. Res. 87: 4148-4154

Chriss, T. C., Caldwell, D. R. (1984a). Universal similarity and the thickness of the viscous sublayer at the ocean floor. J. geophys. Res. 89: 6403-6414

Chriss, T. C., Caldwell, D. R. (1984b). Turbulence spectra from the viscous sublayer and buffer layer at the ocean floor. J. Fluid Mech. 142: 39-55

Comte-Bellot, G. (1976). Hot-wire anemometry. Ann. Rev. Fluid Mech. 8: 209-231

Downing, J. P., Sternberg, R. W., Lister, C. R. B. (1981). New instrumentation for the investigation of sediment suspension processes in the shallow marine environment. Mar. Geol. 42: 19-34

Eckman, J. E., Nowell, A. R. M., Jumars, P. A. (1981). Sediment destabilization by animal tubes. J. mar Res. 39: 361-374

Eckman, J. E., Nowell, A. R. M. (1984). Boundary skin friction and sediment transport about an animal tube mimic. Sedimentology 31: 851-862

Fisher, J. S., Pickral, J., Odum, W. E. (1979). Organic detritus particles: initiation of motion criteria. Limnol. Oceanogr. 24: 529-532

Gilbert, G. C., Murphy, E. C. (1914). Transportation of debris by running water U. S. Geol. Surv. Prof. Paper No. 86, p. $1-263$

Grant, J. (1983). The relative magnitude of biological and physical sediment reworking in an intertidal community. J. mar. Res. 41: 673-689

Grant, J. (1985). A method for measuring horizontal transport of organic carbon over sediments. Can. J. fish. Aquat. Sci. 42: 595-602

Grant, J., Bathmann, U. V., Mills, E. L. (in press). The interaction between benthic diatom films and sediment transport. Estuar. coast. Shelf. Sci. 
Grant, W. D., Boyer, L. F., Sanford, L. P. (1982). The effect of bioturbation on the initiation of sediment motion in noncohesive sediments. J. mar. Res. 40: 659-677

Gust, G. (1982). Tools for oceanic small scale high frequency flows: metal-clad hot wires. J. geophys. Res. 87: 445-447

Horowitz, P., Hill, W. (1980). The art of electronics. Cambridge Univ. Press, Cambridge

Jorgensen, B. B., Revsbeck, N. P. (1985). Diffusive boundary layers and the oxygen uptake of sediments and detritus. Limnol. Oceanogr. 30: 111-122

Jumars, P. A., Nowell, A. R. M. (1984). Fluid and sediment dynamic effects on marine benthic community structure. Am. Zool. 24: 45-55

Kent, J. C., Eaton, A. R. (1982). Stereo photography study of neutral density He-filled bubbles for 3-D motion studies in an engine cylinder. Appl. Optics 21: 904-912

LaBarbera, M., Vogel, S. (1976). An inexpensive thermistor flowmeter for aquatic biology. Limnol. Oceanogr. 21: $750-756$

Landau, L. D., Lifshitz, E. M. (1959). Fluid mechanics. Pergamon, London

Ligrani, P. M., Moffat, R. J. (1979). Artificially thickening a smooth-wall turbulent boundary layer. AIAA (American Institute of Aeronautics and Astronautics) J. 17: 907-910

Ligrani, P. M., Moffat, R. J. (1985). Thermal boundary layers on a rough surface downstream of steps in wall temperature. Boundary-Layer Meteorol. 31: 127-147

Ligrani, P. M., Moffat, R. J., Kays, W. M. (1983). Artificially thickened turbulent boundary layers for studying heat transfer and skin friction on rough surfaces. Trans. Am. Soc. mech. Eng. 105: 146-153

Middleton, G. V., Southard, J. B. (1978). Mechanics of sediment movement. Lect. notes for Short Course No. 3, SEPM (Society of Economic Paleontologists and Mineralogists), Binghamton, New York

Miller, D. C., Jumars, P. A., Nowell, A. R. M. (1984). Effects of sediment transport on deposit feeding: Scaling arguments. Limnol. Oceanogr. 29: 1202-1217

Muschenheim, D. K. (1984). A model of facultative suspension-feeding by spionid polychaetes. In: Wildish, D. J. (ed.) Biology of the sediment-water interface: Report of the St. Andrews Biological Station's 75th Anniversary Workshop. Can. Tech. Rept. Fish. Aquat. Sci. No. 1263, p. $25-27$

Neumann, A. C., Gebelein, C. D., Scoffin, T. P. (1970). The composition, structure and erodability of subtidal mats, Abaco, Bahamas. J. sedim. Petrol. 40: 274-297

Nielsen, P., Green, M. O., Coffey, F. C. (1981). Suspended sediment under waves. Tech. Rept., Univ. of Sydney Geography and Coastal Stud. No. 82 A

Nielsen, P. (1983). Entrainment and distribution of different sand sizes under water waves. J. sedim. Petrol. 53: $423-428$

Nowell, A. R. M., Church, M. (1979). Turbulent flow in a depth-limited boundary laver. J. geophys. Res. 84: 48164824

Nowell, A. R. M., Jumars, P. A., Eckman, J. E. (1981). Effects of biological activity on the entrainment of marine sediments. Mar Geol. 42: 133-153

Nowell, A. R. M., Jumars, P. A. (1984). Flow environments of aquatic benthos. A. Rev. Ecol. Syst. 15: 303-328

Orr, M. H., Grant, W. D. (1982). Acoustic sensing of particles suspended by wave-bottom interactions. Mar Geol. 45: 253-260

Orr, M. H., Rhoads, D. C. (1982). Acoustic imaging of structures and macrofauna in the upper $10 \mathrm{~cm}$ of sediments using a megahertz backscattering system. Mar Geol. 46 . $117-129$

Paola, C. (1983). Flow and skin friction over natural rough beds. Sc. D. thesis, Mass. Inst. Tech., MIT/WHOI-83-18

Peckarsky, B. L. (1983). Use of behavioral experiments to test ecological theory in streams. In: Barnes, J. R., Minshall, G. W. (ed.) Stream ecology: Applications and testing of general ecological theory. Plenum Press, New York, p. 79-98

Penner, S. S., Jerskey, T (1973). Use of lasers for local measurement of velocity components, species densities and temperatures. Ann. Rev. Fluid Mech. 5: 9-30

Raudkivi, A. J. (1976). Loose boundary hydraulics, 2nd ed. Pergamon, New York

Rhoads, D. C., Yingst, J. Y., Ullman, W. (1978). Seafloor stability in central Long Island Sound. Part I. Temporal changes in erodibility of fine-grained sediment. In: Wiley, M. L. (ed.) Estuarine interactions, Academic Press, New York, p. 221-224

Roberson, J. A., Crowe, C. T. (1980). Engineering fluid mechanics, 2nd ed., Houghton Mifflin, Boston

Rubenstein, D. I., Koehl, M. A. (1973). The mechanisms of filter feeding: some theoretical considerations. Am. Nat. 111: 981-994

Schlichting, G. H. (1967). Boundary layer theory. McGrawHill, New York

Steinhart, J. S., Hart. S. R. (1968). Calibration curves for thermistors. Deep Sea Res. 15: 497-503

Streeter, V L., Wylie, E. B. (1979). Fluid mechanics, 7th ed., McGraw-Hill, New York

Taghon, G. L., Nowell, A. R. M., Jumars, P. A. (1984). Transport and breakdown of fecal pellets: Biological and sedimentological consequences. Limnol. Oceanogr. 29: $64-72$

Taylor, P. A., Dyer, K. R. (1976). Theoretical models of flow near the bed and their implications for sediment transport. In: Goldberg, E. D., McCave, I. N., O'Brien, J. J., Steele, J. H. (ed.) The sea, vol. 6, Marine modelling. Wiley, New York, p. 579-601

Tritton, D. J. (1977). Physical fluid dynamics. Van Nostrand Reinhold, Wokingham, England

Vanoni, V A. (1946). Transportation of sediment in suspension. Trans. Am. Soc. Civil Eng. 111: 67-133

Vogel, S. (1981). Life in moving fluids: The physical biology of flow. Willard Grant, Boston

Wildish, D. J., Kristmanson, D. D. (1979). Tidal energy and sublittoral macrobenthic animals in estuaries. J. Fish. Res Bd Can. 36: 1197-1206

Wildish, D. J., Kristmanson, D. D. (1984). Importance to mussels of the benthic boundry layer. Can. J. Fish. Aquat. Sci. 41: $1618-1625$

Williams, G. P. (1970). Flume width and water depth effects in sediment transport experiments. U. S. Geol. Surv. Prof Paper 562-H, p. 1-37

Williams, G. P. (1971). Aids in designing laboratory flumes. U. S. Geol. Surv. Open-File Rept., p. 1-156

Young, R. A., Southard, J. B. (1978). Erosion of fine-grained sediments: seafloor and laboratory experiments. Bull. Geol. Soc. Am. 89: 663-672 